\title{
Die Köche und der Brei
}

\author{
Petra Barzin, Dirk Fox
}

\section{Komplexität}

Softwareentwicklungen sind komplexe Projekte. Das sind sie nicht einmal in erster Linie aufgrund einer großen Zahl von Programmzeilen („lines of code“), auch wenn das in der Regel die offizielle Messlatte zur Bestimmung der Komplexität eines Softwareprojekts ist. Sie sind komplex, weil an ihnen eine große Zahl von Köchen mitmischt - was bekanntlich dazu führt, dass noch der einfachste Brei verdorben wird. Schlimmer noch: Bei der Softwareentwicklung sind nicht einmal alle Köche tatsächlich Köche...

Denn hinter jedem Softwareprojekt steht ein Auftraggeber, der in erster Linie den (von ihm erwarteten) Nutzen der Software sieht - und häufig wenig von Software und deren Entwicklung versteht. Das dient meist weder der Klarheit der Aufgabenstellung noch einer realistischen Einschätzung des für die Entwicklung erforderlichen Aufwands. Am wenigsten aber dient es der Sicherheit der späteren Anwendung - ein Thema, dass der Auftraggeber in der operativen Hektik oder Euphorie meist unten in der Prioritätenskala einsortiert.

Dem Auftraggeber sitzt wiederum nicht der Entwickler, sondern ein Software-"Architekt", -"Planer" oder -"Designer" gegenüber, der die Anforderungen des Auftraggebers präzisieren und in ein Umsetzungskonzept überführen muss. Als „Designer“ ist er stolz auf seine kreativen Lösungen - und daher oft wenig motiviert, nach „best practice“-Ansätzen Ausschau zu halten oder sich an bewährten Konzepten Anderer zu orientieren. Keine gute Voraussetzung, um die keineswegs triviale Herausforderung eines „sicheren Designs“ zu meistern, ohne die schlechten Erfahrungen Anderer zu wiederholen.

Das Umsetzungskonzept landet schließlich beim Entwickler. Da er sportliche Zeitvorgaben erhält, wird er versuchen, sich auf die Kernfunktionalität zu konzentrieren und „Schnickschnack“ auf den eingeplanten (aber tatsächlich nie verbleibenden) zeitlichen „Sicherheitspuffer“ zu verschieben. $\mathrm{Zu}$ diesem "Schnickschnack“ wird er meist auch den Umgang mit unerwarteten Eingaben eines Nutzers zählen. Fatal, denn das häufigste Einfallstor für Angriffsprogramme sind die Input/Output-Schnittstellen: Pufferüberläufe und Cross-Site-Scripting bei Dateneingaben und Replay- oder Man-inthe-Middle-Angriffe bei Kommunikationsverbindungen.

Bleibt der Tester. Sofern er überhaupt mit einem Testplan arbeitet, wird er seine Tests auf die Prüfung der spezifizierten Funktionalität konzentrieren. Schließlich ist es auch schwierig, das Programmverhalten bei unerwartetem Anwenderverhalten (also z. B. dem eines Angreifers) zu prüfen - das liegt in der Natur des „Unerwarteten“. Zwar kann „Fuzzing"1 helfen; oft sind solche modernen Hilfsmittel für die Suche nach sicherheitsrelevanten Fehlern Testern aber nicht bekannt. Schließlich findet das Testen seine Grenze in der Prüfung seltener Sonderfälle wie unwahrscheinlichen Kombinationen von Randbedingungen oder Fehlerhäufungen.

\section{Abhängigkeit}

Verstärkt wird das Komplexitätsproblem durch die - dank zahlreicher unterschiedlicher Kommunikationsschnittstellen - ständig zunehmenden Verknüpfungsmöglichkeiten unterschiedlicher Anwendungen einerseits und der Modularisierung von Softwarelösungen andererseits. Dadurch entstehen kaum noch überschaubare Abhängigkeiten innerhalb und zwischen Softwareprodukten, die dazu führen können, dass ein seltener Einzelfehler gleich zahlreiche Anwendungen betrifft. Auch die grundsätzlich - nicht zuletzt aus Sicherheitsperspektive begrüßenswerte Wiederverwendung von Softwareteilen, die durch die Verbreitung von Open Source begünstigt wird, verstärkt die Abhängigkeit: Ein einziger Fehler in einer Programmroutine kann leicht Hunderte von Implementierungen betreffen. ${ }^{2}$

\footnotetext{
${ }^{1}$ Siehe Fox, Gateway, DuD 12/2006, S. 795.

${ }^{2}$ So geschehen am 30.09.2003, als ein veröffentlichter Fehler im ASN.1-Parser von OpenSSL gleich zahlreiche kommerzielle Produkte betraf.
}

\section{Zu diesem Heft}

Abhängigkeit und Komplexität sind nicht einfach nur zwei Fehlerursachen unter vielen. Die andauernde Komplexitätszunahme hat, wie die Freiburger InformatikProfessorin Britta Schinzel 2001 schrieb, „bisher alle qualitätssichernden Innovationen" überholt - mit der Konsequenz, dass „die Zuverlässigkeit langsamer als die Innovationen" wächst. ${ }^{3}$ Eine ernüchternde Feststellung, steigt mit der Komplexität der Systeme doch zugleich unsere Abhängigkeit von deren Funktionieren. Kein Wunder also, dass sich das Thema „Entwicklung sicherer und zuverlässiger Software" wachsender Aufmerksamkeit erfreut - und sich ein Jahr nach dem DuD-Schwerpunktheft 10/2006 nun problemlos ein zweites füllen ließ.

Immerhin: Die Lösungsansätze und Vorschläge sind viel versprechend. Zwei Trends sind erkennbar: Einerseits wird der Hebel an der „Entwicklungsdisziplin“ angesetzt. So hat das Projekt secologic zahlreiche „Goldene Regeln“ für die unterschiedlichen, an einem Software-Entwicklungsprozess beteiligten Parteien hervor gebracht, die Rosemaria Giesecke und Stefan Fünfrocken in ihrem Beitrag vorstellen. Und das iSQI hat ein Qualifizierungszertifikat 'Certified Professional for Secure Software Engineering' initiiert. Andererseits wird versucht, die Überprüfung von Software auf sicherheitskritische Fehler zumindest teilweise $\mathrm{zu}$ automatisieren. Martin Johns und Daniel Schreckling stellen dazu die aktuelle Entwicklung im Bereich der automatisierten Code-Analyse vor.

Petra Barzin konzentriert sich in ihrem Beitrag auf Design-Fehler - die (hinsichtlich der nachträglichen Beseitigung) teuerste Klasse sicherheitsrelevanter Bugs. Und im Gateway erläutert sie das „Reifegradmodell" für sichere Software.

\footnotetext{
${ }^{3}$ http://mod.iig.uni-freiburg.de/cms/fileadmin/ publikationen/online-publikationen/kompl.pdf
} 